\title{
Turbulent flow around a square cylinder at Reynolds number 22000: a DNS study
}

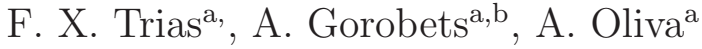 \\ ${ }^{a}$ Heat and Mass Transfer Technological Center, Technical University of Catalonia \\ ETSEIAT, c/ Colom 11, 08222 Terrassa, Spain \\ ${ }^{b}$ Keldysh Institute of Applied Mathematics, 4A, Miusskaya Sq., Moscow 125047, Russia
}

\section{Abstract}

The turbulent flow around a square cylinder at Reynolds number 22000 (based on the cylinder diameter and the inflow velocity) is studied by means of direct numerical simulation. An overview of the numerical methods and the methodology used to verify the simulation is presented with special emphasis to determine the proper domain size and time-integration period. Then, the time-averaged flow results and turbulent statistics are discussed together with available experimental data showing a fairly good agreement. Finally, frequency analysis of velocity samples is used to analyze both the Kelvin-Helmholtz vortical structures produced by the flow separation at the leading edge of the cylinder and the Von Kármán vortex shedding in the wake region. The former are observed more downstream compared with the experiments suggesting that transition to turbulence may occur later. However, comparison of the turbulent statistics in the near wall region indicates that transition is being well captured.

\footnotetext{
*Tel: +34 9373981 92; Fax: +34 937398101.

Email addresses: xavi@cttc.upc.edu (F. X. Trias), andrey@cttc.upc.edu

(A. Gorobets), oliva@cttc.upc.edu (A. Oliva)
} 
Key words:

DNS, square cylinder, turbulence, vortex shedding

\section{Introduction}

The flow around a square cylinder constitutes a canonical configuration to study the flow around bluff bodies. Except for very low Reynolds numbers, $R e$ (based on the inflow velocity and the cylinder width), the flow separates from the upstream corners and vortices are formed. The asymmetric shedding of these vortices into the wake induces forces on the cylinder leading to structural vibration. Such a vibration is termed as Vortex-Induced Vibration (VIV). This fluid excitation forms a potent source of fatigue and flow-induced noise for many engineering applications. Towering structures, skyscrapers, marine riser pipes, long-spanned bridges and wires are examples thereof. Hence, the flow around a square cylinder is a canonical test-case of great interest not only to study the VIV phenomenon but also bluff body aerodynamics. Therefore, this and similar configurations have been the subject of numerous experimental and numerical studies in the last decades.

The majority of the research on flow around cylindrical objects has been carried out for circular cylinders [1-12]. In this regard, since the first ex-

perimental results were published in the mid-90s [5], the configuration at $R e=3900$ has attracted the attention of many researchers. It is at the crossroad between experimental and computational capabilities; therefore, it also motivated many numerical studies. For instance, several direct numerical simulations (DNS) studies [6-8] have been published for this configuration providing a good agreement with experimental results. Moreover, it has 
also been extensively used for benchmarking purposes to validate turbulence models and numerical methods [9-12].

The flow around cylinders with rectangular cross sections has also been widely studied in the last decades. The main difference respect to the circular cylinder is that the flow separates from the sharp corners; therefore, separation points are fixed whereas for circular cylinders they are time-dependent. In this respect, many experimental studies can be found in the literature [1322]. Regarding numerical studies the state-of-the-art is far from being satisfactory. Very accurate numerical studies can be found in the laminar regime. For instance, a set of $2 \mathrm{D}$ simulations for a square cylinder at $R e \leq 300$ were performed in [23]. The same configuration was studied for different angles of incidence and $40 \leq R e \leq 200$ in [24]. The transition from 2D to 3D shedding flow was studied by the same authors in [25] reaching the conclusion that it occurs between $R e=150$ and $R e=200$. This aspect was also studied in [26] finding that this transition takes place between $R e=150$ and $R e=175$. More recent studies in the laminar regime can be found in [27, 28], for instance. For $R e=500$, the highest $R e$-number studied in [25, 26], the shear layer remains stable and rolls up to form the Von Kármán vortex street in the wake region. The first Kelvin-Helmholtz instability in the shear layer appears at $R e \approx 1000$. This was observed in [22] where large-eddy simulations (LES) at $R e \leq 2000$ and experiments for a wide range of Re-numbers were carried out. They also concluded that Kelvin-Helmholtz structures are present for a large $R e$-number range until the transition to turbulence takes place too close to the upper corner. Within this range, the configuration at $R e=22000$ has been considered in many research works. It was selected as test-case in some 
workshops about turbulence modeling in the mid-90s [29, 30]. However, most of the numerical studies have been performed using RANS and LES modeling techniques $[20,29-33]$ while attempts to perform DNS [34, 35] are quite scarce and limited to relatively coarse meshes and short integration periods. Hence, the experimental results by Lyn and Rodi [18] and Lyn et al. [19] have been usually taken as a reference. Other experimental results for this configuration can also be found in the literature [13, 14, 22]. Recently, Minguez et al. [20] published detailed experimental results of the near-wall region together with a LES simulation. However, since this flow configuration is used for benchmarking purposes to validate turbulence models and numerical methods the availability of accurate numerical results is of extreme importance. This is addressed in the present paper where DNS results are presented and compared with experimental results of Lyn et al. [19] and Minguez et al. [20]. To assess the quality of the results, the influence of numerical and physical parameters have been carefully studied here. For instance, compared with previous LES/RANS numerical studies both cross-stream and stream-wise directions have been increased in order to obtain results independent of the domain size. Moreover, it has also been found that the total integration period used in previous numerical studies was insufficient to obtained fully converged results. These finding can provide guidance for future numerical studies. Moreover, it should be noted that apart from giving insights into the physics of turbulent flows around bluff bodies, the aim of this work is to provide reference data [36] for this canonical configuration and not to reproduce any particular experimental set-up. For instance, the free-stream turbulent intensity and the blockage of the experiments are examples of factors that 


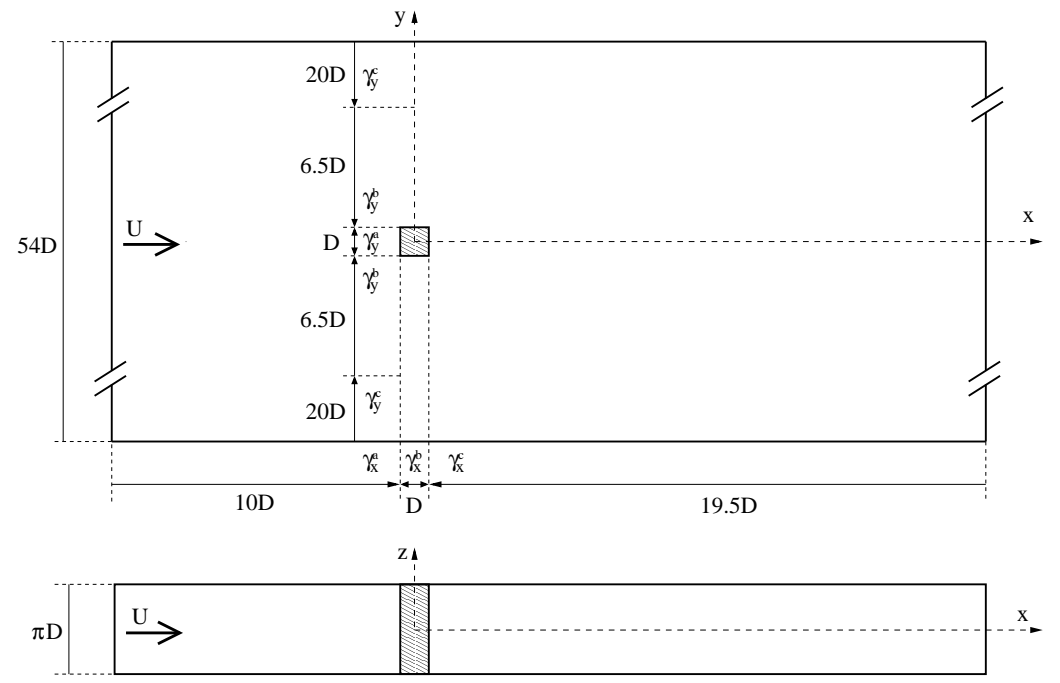

Figure 1: Schema of the square cylinder problem and details about the grid spacing (size of zones and concentration factors; arrows indicate the grid refinement direction).

can significantly affect the results and are not considered here. Therefore, comparison with the above-mentioned experiments should be viewed with caution.

The rest of the paper is arranged as follows. In the next section, the governing equations and the problem definition are described together with an overview of the numerical methods. Then, the methodology to verify the simulation is presented in Section 3. The core of the results is in Section 4. Firstly, the main features of the time-averaged flow are discussed with a direct comparison with previous experimental results. Then, the discussion focuses on the flow dynamics; namely, turbulent statistics are presented together with the frequency analysis of velocity samples at different flow locations in the shear layer zone and in the wake region. Finally, relevant results are summarized and conclusions are given in the last section. 


\section{Governing equations and numerical methods}

The incompressible Navier-Stokes (NS) equations in primitive variables are considered

$$
\frac{\partial \mathbf{u}}{\partial t}+(\mathbf{u} \cdot \nabla) \mathbf{u}=\nu \nabla^{2} \mathbf{u}-\nabla p ; \quad \nabla \cdot \mathbf{u}=0
$$

where $\mathbf{u}=(u, v, w)$ is the velocity field, $p$ represents the kinematic pressure and $\nu$ is the kinematic viscosity. A schema of the problem under consideration is displayed in Figure 1. In this case, the dimensionless Reynolds number, $R e=U D / \nu$, is based on the inflow velocity, $U$, and the cylinder width, $D$. The dimensions of the computational domain are $30.5 D \times 54 D \times \pi D$ in the stream-wise, cross-stream and span-wise direction, respectively. The upstream face of the cylinder is located at $10 D$ from the inflow and centered in the cross-stream direction. A detailed discussion about the determination of the domain size and grid spacing is given in the next section. The origin of coordinates in placed at the center of the cylinder. Regarding the boundary conditions, a constant velocity profile, $\mathbf{u}=(U, 0,0)$, is imposed at the inflow, convective boundary conditions are used at the outflow, $\partial \mathbf{u} / \partial t+U \partial \mathbf{u} / \partial x=0$, and Neumann boundary condition is adopted in the cross-stream direction for all velocity components, $\partial \mathbf{u} / \partial y=0$. Global mass conservation may be not exactly preserved after imposing such boundary conditions. Here, it is forced by means of a minor correction (a constant stream-wise velocity profile many orders of magnitude lower than $U$ ) at the outflow conditions. Periodic boundary conditions are imposed in the span-wise direction. Finally, no-slip boundary conditions are imposed at the surface of the cylinder. Hereafter,

the results are presented in dimensionless form where the reference length, 
velocity, time and kinematic pressure are $D, U, D / U, U^{2} / 2$, respectively.

The incompressible NS equations (1) are discretized on a staggered Cartesian grid using a fourth-order symmetry-preserving discretization [37]. Shortly, the temporal evolution of the spatially discrete staggered velocity vector, $\mathbf{u}_{h}$, is governed by the following operator-based finite-volume discretization of Eqs.(1)

$$
\boldsymbol{\Omega} \frac{d \mathbf{u}_{h}}{d t}+\mathbf{C}\left(\mathbf{u}_{h}\right) \mathbf{u}_{h}+\mathbf{D} \mathbf{u}_{h}-\mathbf{M}^{t} \mathbf{p}_{h}=\mathbf{0}_{h},
$$

where the discrete incompressibility constraint is given by $\mathbf{M} \mathbf{u}_{h}=\mathbf{0}_{h}$ and the subscript $h$ refers to discrete vectors. The diffusive matrix, $\mathbf{D}$, is symmetric and positive semi-definite; it represents the integral of the diffusive flux, $-\nu \nabla \mathbf{u} \cdot \boldsymbol{n}$, through the faces. The diagonal matrix, $\boldsymbol{\Omega}$, describes the sizes of the control volumes and the approximate, convective flux is discretized as in [37]. The resulting convective matrix, $\mathbf{C}\left(\mathbf{u}_{h}\right)$, is skew-symmetric, i.e.

$$
\mathbf{C}\left(\mathbf{u}_{h}\right)=-\mathbf{C}^{t}\left(\mathbf{u}_{h}\right) .
$$

In a discrete setting, the skew-symmetry of $\mathbf{C}\left(\mathbf{u}_{h}\right)$ implies that

$$
\mathbf{C}\left(\mathbf{u}_{h}\right) \mathbf{v}_{h} \cdot \mathbf{w}_{h}=\mathbf{v}_{h} \cdot \mathbf{C}^{t}\left(\mathbf{u}_{h}\right) \mathbf{w}_{h}=-\mathbf{v}_{h} \cdot \mathbf{C}\left(\mathbf{u}_{h}\right) \mathbf{w}_{h},
$$

for any discrete velocity vectors $\mathbf{u}_{h}$ (if $\mathbf{M} \mathbf{u}_{h}=\mathbf{0}_{h}$ ), $\mathbf{v}_{h}$ and $\mathbf{w}_{h}$. Then, the evolution of the discrete energy, $\left\|\mathbf{u}_{h}\right\|^{2}=\mathbf{u}_{h} \cdot \boldsymbol{\Omega} \mathbf{u}_{h}$, is governed by

$$
\frac{d}{d t}\left\|\mathbf{u}_{h}\right\|^{2}=-2 \mathbf{u}_{h} \cdot \mathbf{D} \mathbf{u}_{h}<0,
$$

where the convective and pressure gradient contributions cancel because of Eq.(3) and the incompressibility constraint, $\mathbf{M} \mathbf{u}_{h}=\mathbf{0}_{h}$, respectively. Therefore, even for coarse grids, the energy of the resolved scales of motion is 
convected in a stable manner, i.e. the discrete convective operator transports energy from a resolved scale of motion to other resolved scales without dissipating any energy, as it should be from a physical point-of-view. For a detailed explanation, the reader is referred to [37].

The governing equations are integrated in time using a classical fractional step projection method [38]. Namely, the solution of the unsteady NavierStokes equations is obtained by first time-advancing the velocity field, $\mathbf{u}^{n}$, without regard for its solenoidality constraint, then recovering the proper solenoidal velocity field, $\mathbf{u}^{n+1}\left(\nabla \cdot \mathbf{u}^{n+1}=0\right)$. For the temporal discretization, a fully second-order explicit one-leg scheme is used for both the convective and diffusive terms [39]. Thus, the resulting fully-discretized problem reads

$$
\frac{(\kappa+1 / 2) \mathbf{u}_{h}^{p}-2 \kappa \mathbf{u}_{h}^{n}+(\kappa-1 / 2) \mathbf{u}_{h}^{n-1}}{\Delta t}=\mathbf{R}\left((1+\kappa) \mathbf{u}_{h}^{n}-\kappa \mathbf{u}_{h}^{n-1}\right),
$$

where $\mathbf{R}\left(\mathbf{u}_{h}\right)=-\mathbf{C}\left(\mathbf{u}_{h}\right) \mathbf{u}_{h}-\mathbf{D} \mathbf{u}_{h}$ and $\mathbf{u}_{h}^{p}$ is a predictor velocity that can be directly evaluated from the previous expression. The time-integration parameter, $\kappa$, is computed to adapt the linear stability domain of the timeintegration scheme to the instantaneous flow conditions in order to use the maximum time-step, $\Delta t$, possible. For further details about the time-integration method the reader is referred to [39]. Finally, $\mathbf{u}_{h}^{p}$ must be projected onto a divergence-free space,

$$
\mathbf{u}_{h}^{n+1}=\mathbf{u}_{h}^{p}+\Omega^{-1} \mathbf{M}^{t} \tilde{\mathbf{p}}_{h}^{n+1}
$$

by adding the gradient of the pseudo-pressure, $\tilde{\mathbf{p}}_{h}=\Delta t /(\kappa+1 / 2) \mathbf{p}_{h}$, satisfying the following Poisson equation

$$
\mathbf{L} \tilde{\mathbf{p}}_{h}^{n+1}=\mathbf{M} \mathbf{u}_{h}^{p} \quad \text { with } \quad \mathbf{L}=-\mathbf{M} \mathbf{\Omega}^{-1} \mathbf{M}^{t}
$$


where the discrete Laplacian operator, $\mathbf{L}$, is represented by a symmetric negative semi-definite matrix. For details about the numerical algorithms and the parallel Poisson solver the reader is referred to [40]. Notice that pressure is not considered in the prediction step (6). This approach combined with an implicit treatment of viscous terms may lead to first-order errors in time [41]. In that case, second-order accuracy can be achieved by using pressure correction methods [42] or using proper modifications of the boundary conditions (see [43] and references therein). However, in our case the time-integration scheme is fully explicit; therefore, the second-order temporal accuracy of the scheme is not affected in the projection step. Furthermore, since a staggered arrangement is used, ignoring pressure in the prediction step produces exactly the same results as a pressure correction method [44]. On the other hand, on staggered grids with prescribed velocity boundary conditions, as in our case, the incompressibility condition occurs naturally and no specific boundary condition for the discrete pressure field, $\mathbf{p}_{h}$, needs to be specified as pointed out in [45]. Nevertheless, for practical purposes Neumann boundary conditions are prescribed for $\mathbf{p}_{h}$. Regarding the verification of the code the reader is referred, for instance, to [46]. In addition, rigorous comparison with accurate previous numerical studies [23-25] of the flow around a square cylinder has been used to verify the code for this configuration. The verification process of the DNS simulation carried out in this work is addressed in the next section. 

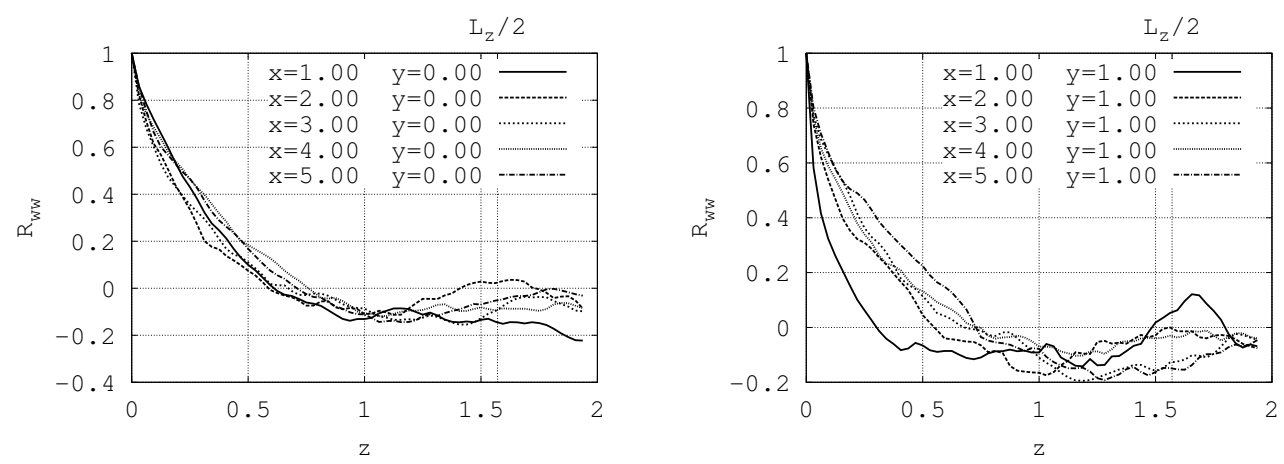

Figure 2: Two-point correlations of the span-wise velocity, $w$, at five monitoring locations. In this case, $L_{z}=\pi$ corresponds to the size in the span-wise direction for MeshA (see Table 1).

\section{Verification of the simulation}

Since no subgrid-scale model is used, the grid resolution and the time step must be fine enough to capture well all the relevant turbulent scales. Moreover, the domain in the periodic direction, $L_{z}$, must be long enough, keeping an adequate mesh resolution, $\Delta z$, to ensure that numerical solution is not affected. Besides, the cylinder must be far enough from the boundary conditions imposed in both the stream-wise and cross-stream directions. Finally, the starting time for averaging and the time integration period must also be long enough to evaluate the flow statistics properly.

In a preliminary simulation, we have used a $708 \times 708 \times 128($ MeshB) Cartesian staggered grid to cover the computational domain (see Table 1 for details). In this case, spatial discretization is second-order accurate [37] and the span-wise length is set to $L_{z}=4$. This is the same $L_{z}$ as the original problem proposed in the ERCOFTAC workshops [29, 30] and thereafter used for most of the numerical studies. Compared with previous LES cal- 
culations presented in [47] where $L_{z}=2$, in those workshops the domain in the span-wise direction was extended to $L_{z}=4$ to allow the correct formation of 3D structures. In any case, this must be long enough to ensure that turbulent fluctuations are uncorrelated at a separation of one half-period, $L_{z} / 2$. Figure 2 displays span-wise two-point correlation analysis of the spanwise velocity component, $R_{w w}$, at five different $(x, y)$-locations. For all cases, the correlation values fall to zero for separations lower than one half-period. Similar results are obtained for other $(x, y)$-locations and variables. Actually, results show that a shorter span-wise length suffices. Hence, in the view of lower cost and better grid resolution in that direction, $L_{z}=\pi$ has been used for the finest grid. Regarding the stream-wise and the cross-stream directions the physical domain corresponds to the problem originally proposed in the ERCOFTAC workshops $[29,30]$ except for the buffers zones of $20 D$ added at the top and bottom (see Figure 1). In a set of preliminary simulations it was observed that the size of the domain in the cross-stream direction have a relevant influence on the results probably due to some blockage effects; therefore, it motivated the proposed approach. Despite these additional areas are quite large they 'only' represent $\approx 18 \%$ of the total number of control volumes. As mentioned above, the stream-wise direction corresponds to the original problem proposed in the ERCOFTAC workshops $[29,30]$, i.e. $L_{x}=27 D$ with the upstream face of the cylinder located at 6.5D. Additional tests with meshes equivalent to MeshB but increasing this distance up to $10 D$ and 13.5D revealed small variations. Since no difference was observed between using $10 D$ and $13.5 D$, for MeshA the upstream face of the cylinder is located at $10 \mathrm{D}$. No significant influence was observed by increasing the distance of 


\begin{tabular}{|c|c|c|c|c|c|c|c|c|c|c|c|c|c|c|c|c|}
\hline & & Case & $N_{x}$ & $N_{y}$ & $N_{z}$ & $L_{x}$ & $L_{y}$ & $L_{z}$ & $\gamma_{x}^{a}$ & $\gamma_{x}^{b}$ & $\gamma_{x}^{c}$ & $\gamma_{y}^{a}$ & $\gamma_{y}^{b}$ & $\gamma_{x}^{c}$ & \multirow[b]{4}{*}{$\begin{array}{l}\text { Total } \\
\text { time }\end{array}$} & \multirow[b]{4}{*}{$\begin{array}{c}\text { Average } \\
\text { time }\end{array}$} \\
\hline & & MeshA & 1272 & $2 \quad 1174$ & 2163 & 30.5 & 54 & $\pi$ & 2.2 & 0.5 & 2.0 & 0.5 & 2.0 & 1.75 & & \\
\hline & & MeshB & 708 & 708 & 128 & 27 & 54 & 4 & 2.2 & 0.5 & 2.0 & 0.5 & 2.0 & 1.75 & & \\
\hline Case & $(\Delta x)_{\min }$ & \multicolumn{2}{|c|}{$(\Delta y)_{\min }$} & $(\Delta x)_{\min }^{+}$ & $(\Delta y)_{\min }^{+}$ & \multicolumn{3}{|c|}{$(\Delta x)_{\max }$} & \multicolumn{2}{|c|}{$(\Delta y)_{\max }$} & \multicolumn{2}{|c|}{$\Delta z$} & & $\Delta t$ & & \\
\hline MeshA & $1.89 \times 10^{-3}$ & \multicolumn{2}{|c|}{$3 \quad 1.44 \times 10^{-3}$} & $\lesssim 0.56$ & $\lesssim 0.15$ & \multicolumn{3}{|c|}{$7.83 \times 10^{-2}$} & \multicolumn{2}{|c|}{$3.46 \times 10^{-1}$} & \multicolumn{2}{|c|}{$1.454 \times 10^{-2}$} & \multicolumn{2}{|c|}{$9.70 \times 10^{-4}$} & $\approx 605$ & $\approx 505$ \\
\hline MeshB & $3.13 \times 10^{-3}$ & \multicolumn{2}{|c|}{${ }^{3} \quad 2.39 \times 10^{-3}$} & $\lesssim 0.93$ & $\lesssim 0.25$ & \multicolumn{3}{|c|}{$1.45 \times 10^{-1}$} & \multicolumn{2}{|c|}{$6.43 \times 10^{-1}$} & \multicolumn{2}{|c|}{$3.125 \times 10^{-2}$} & \multicolumn{2}{|c|}{$1.71 \times 10^{-3}$} & $\approx 300$ & $\approx 200$ \\
\hline
\end{tabular}

Table 1: Physical and numerical simulation parameters.

the obstacle from the outflow.

Once the physical parameters are controlled, the grid resolution and the time step need to be determined. Grid spacing in the period z-direction is uniform whereas the wall-normal points are distributed using piece-wise hyperbolic-tangent functions. For instance, the distribution of points in the $x$-direction in the region corresponding with the obstacle, i.e. $-1 / 2 \leq x \leq$ $1 / 2$, is given by

$$
x_{k}=x_{0}^{b}+\frac{L_{x}^{b}}{2}\left(1+\frac{\tanh \left\{\gamma_{x}^{b}\left(2(k-1) / N_{x}^{b}-1\right)\right\}}{\tanh \gamma_{x}^{b}}\right), \quad k=1, \ldots, N_{x}^{b}+1 .
$$

where the starting point and the region length are $x_{0}^{b}=-1 / 2$ and $L_{x}^{b}=1$, respectively. Notice that in this region, mesh is refined in both directions. Grid refinement formula needs to be properly adapted for those areas where the mesh is refined only in one direction (see Figure 1 and Table 1, for details). For instance, the grid points in the region downstream the obstacle, i.e. $0.5 \leq$ $x \leq 20$, are distributed as follows

$$
x_{k}=x_{0}^{c}+\frac{L_{x}^{c}}{2}\left(1+\frac{\tanh \left\{\gamma_{x}^{c}\left((k-1) / N_{x}^{c}-1\right)\right\}}{\tanh \gamma_{x}^{c}}\right), \quad k=1, \ldots, N_{x}^{c}+1
$$


In this case, the starting point and the region length are $x_{0}^{c}=0.5$ and $L_{x}^{c}=2(20-0.5)=39$, respectively. Then, the concentration factors, $\gamma_{x}^{a}$, $\gamma_{x}^{b}$ and $\gamma_{x}^{c}$ are determined from the criterion proposed in [46]; namely, the flow gradients on the computational space are minimized for a set of representative instantaneous maps. Then, the number of grid points follows straightforwardly by imposing that $N_{x}^{a}+N_{x}^{b}+N_{x}^{c}=N_{x}$ and that the sizes of two consecutive control volumes corresponding to different areas are equal. The grid points in the $y$-direction are distributed in the same way. The values of the concentration factors together with other relevant simulation parameters can be found in Table 1. Furthermore, the exact grid point distribution can be found in [36].
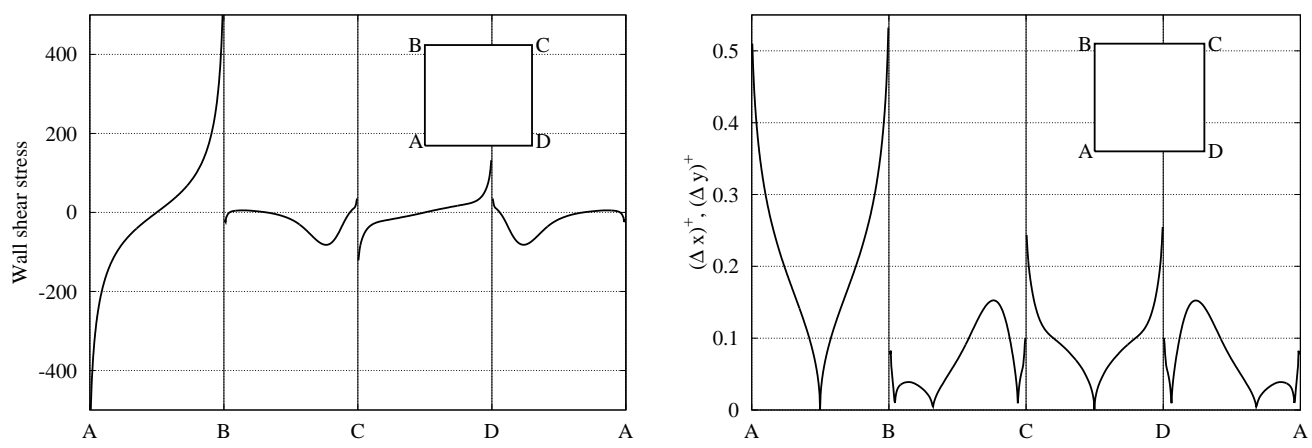

Figure 3: Time-averaged wall-shear stress (left) and location of the first grid point in wall-units (right) for MeshA.

The region most sensitive to the grid resolution is near the obstacle. Figure 3 displays the location of the first grid point in wall-units for the MeshA. Note that the friction velocity, $u_{\tau}$, is computed with the local wall-shear stress. This value falls down to values smaller than unity for the four obstacle walls indicating that the grid is fine enough. In turbulent regions, the 

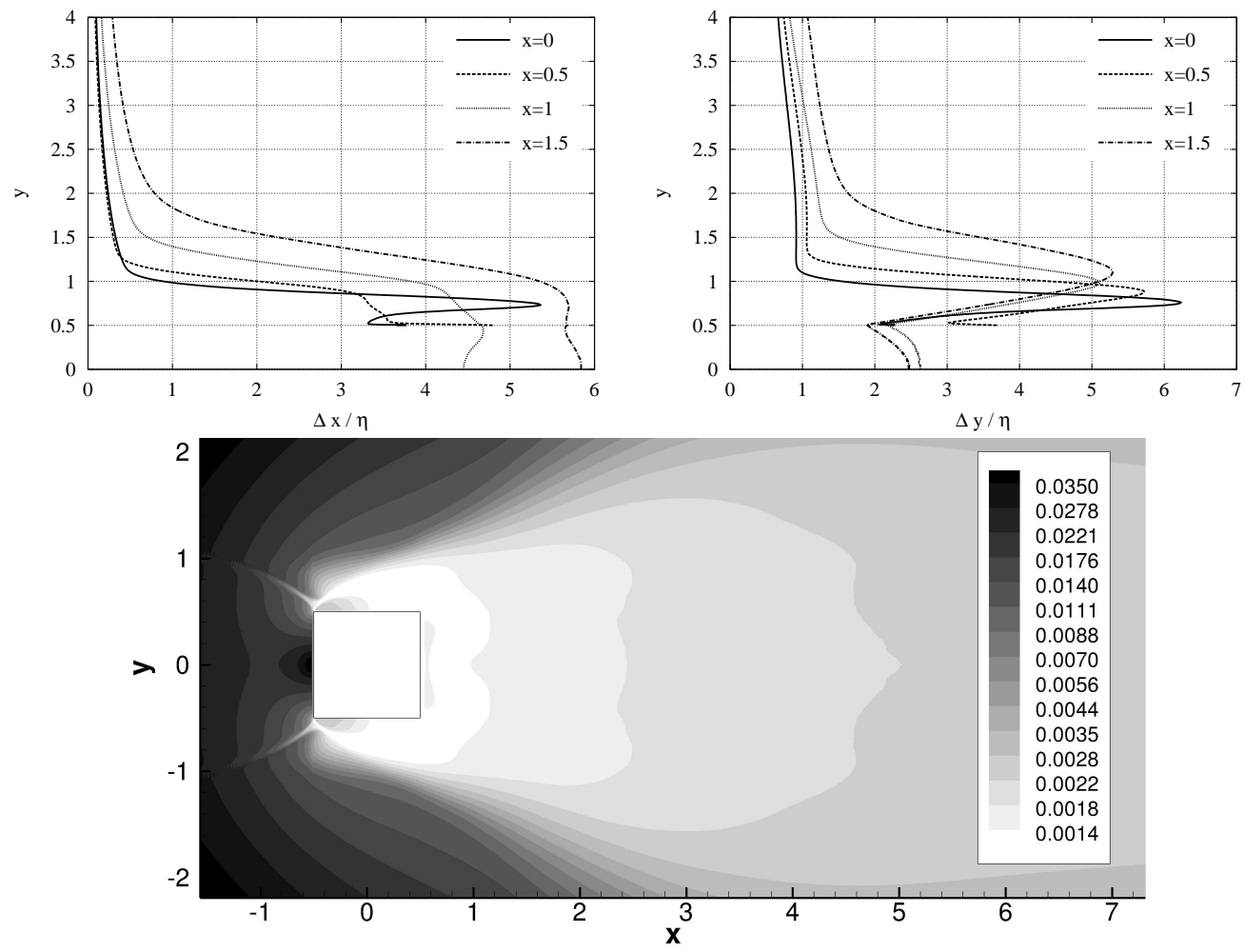

Figure 4: Top: ratio between the stream-wise (left) and the cross-stream (right) grid spacing and the Kolmogorov length scale, $\eta$, at different locations. Bottom: Kolmogorov length scale, $\eta$, around the square cylinder. Results obtained for MeshA.

smallest resolved length scale is required to be $\mathcal{O}(\eta)$ where $\eta=\left(\nu^{3} /\langle\epsilon\rangle\right)^{1 / 4}$ is the Kolmogorov length scale, $\langle\epsilon\rangle=2 \nu\left\langle S^{\prime}: S^{\prime}\right\rangle$ is the time-averaged local dissipation of turbulent kinetic energy and $S^{\prime}=1 / 2\left(\nabla \mathbf{u}^{\prime}+\left(\nabla \mathbf{u}^{\prime}\right)^{t}\right)$ is the fluctuating rate-of-strain tensor. Figure 4 (top) displays the ratio between the stream-wise, $\Delta x$, and the cross-stream, $\Delta y$, grid spacings and $\eta$. As expected, peaks are observed in the shear layer zone where values of $\langle\epsilon\rangle$ are higher; therefore, the smallest values of the Kolmogorov length scale, $\eta$, are observed in this region (see Figure 4, bottom). These peaks tend to 
increase for more downstream positions because the mesh becomes coarser. These values are similar to the resolution requirements suggested to obtain accurate first- and second-order statistics [48, 49]. They follow from the criterion that most of the dissipation is being capture. Therefore, grid spacings equal or smaller than $\eta$ are considered too stringent because the Kolmogorov length scale is at the far end of the dissipative range. In this regard, a recent work [50] has shown that most of the dissipation in a turbulent channel flow occurs at scales greater than $30 \eta$. Even more recently, values of $\eta \lesssim 3.5$ sufficed to obtain very accurate results for a DNS simulation of a turbulent square duct flow [51] using the same numerics than here. Regarding the time-step, $\Delta t$, it follows from the self-adaptive stability criterion proposed in [39]. The resulting time-step, $\Delta t$, is sufficiently lower than the smallest relevant temporal scale. For instance, for MeshA, $\Delta t=9.7 \times 10^{-4}$ (see Table 1) is significantly smaller than the smallest Kolmogorov time scale, $\tau_{\eta}=(\nu /\langle\epsilon\rangle)^{1 / 2} \gtrsim 6.8 \times 10^{-3}$. Starting from an initial velocity field at rest (except for a small random perturbation to trigger the three-dimensionality), approximately 605 time-units have been computed. The last 505 time-units has been used to obtained the time-averaged solution. This approximately corresponds with 67 shedding cycles. In this regard, an averaging period of 13 vortex shedding was suggested in [30]. Most of subsequent LES works followed this recommendation: namely, Rodi [31], Ochoa \& Fueyo [33] and Minguez et al. [20] computed time-averaged results over 10, 12 and 13 shedding cycles, respectively. Sohankar et al. [32] used a longer integration period of about 20 shedding cycles. In this work, we observed that significantly longer time-integration periods are required to obtain accurate results for 
all quantities of interest. The temporal convergence study displayed in Figure 5 is a clear example thereof. For instance, accurate predictions (with differences $\lesssim 0.01$ ) of the drag coefficient, $\left\langle C_{D}\right\rangle$, are only obtained after $\gtrsim 40$ shedding cycles. Other quantities show a similar convergence behavior. Hereafter the numerical results obtained with the MeshA (see Table 1, for details) are referred as the DNS solution.
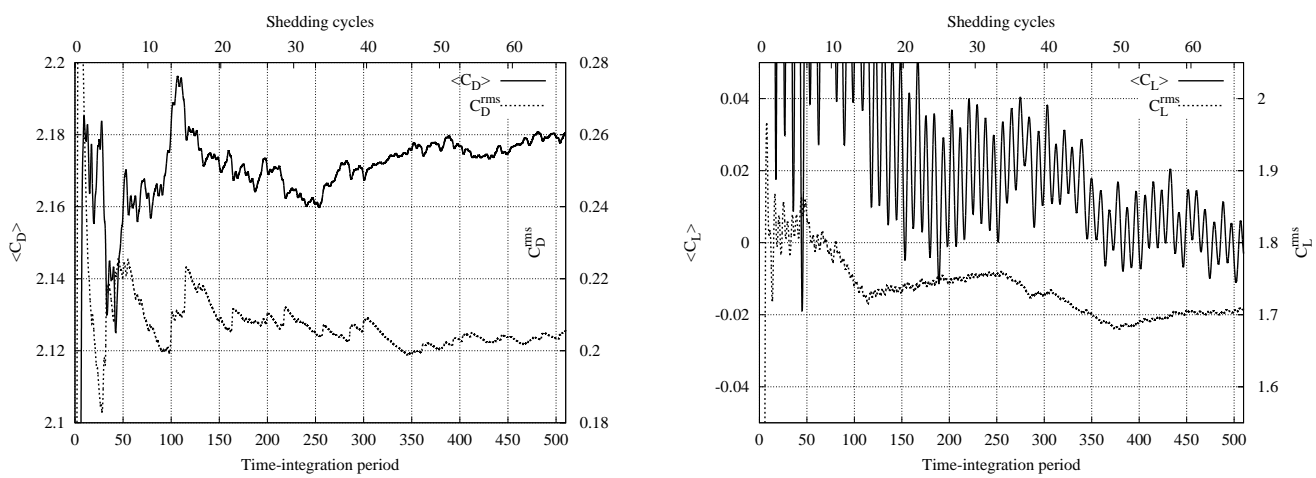

Figure 5: Temporal convergence study. Left: $\left\langle C_{D}\right\rangle$ and $C_{D}^{r m s}$. Right: $\left\langle C_{L}\right\rangle, C_{L}^{r m s}$ (right). Results are obtained with the last time-units, i.e. $\left\langle C_{D}\right\rangle=1 /\left(t_{\text {end }}-t\right) \int_{t}^{t_{\text {end }}} C_{D}(t) d t$ where $t_{\text {end }}=605($ see Table 1$)$.

\section{Results and discussion}

\subsection{Time-averaged flow}

Averages over the three statistically invariant transformations (time, $z$ direction and central plane symmetry) are carried out for all the fields. The standard notation $\langle\cdot\rangle$ is used to denote this averaging procedure. The timeaveraged pressure field and the streamlines of the average flow are displayed in Figure 6. The laminar upstream flow impinges the front wall of the cylinder. Consequently, this is a region with high pressure values. Then, the 


\begin{tabular}{lcccccc} 
Case & $R e / 10^{3}$ & $S t$ & $\left\langle C_{D}\right\rangle$ & $C_{D}^{r m s}$ & $C_{L}^{r m s}$ & $l_{R}$ \\
\hline \hline DNS & 22 & 0.132 & 2.18 & 0.205 & 1.71 & 1.04 \\
\hline Minguez et al. $[20]$ & 21.4 & 0.130 & 2.1 & - & - & - \\
Lyn et al. $[18$, 19] & 21.4 & 0.133 & 2.1 & - & - & - \\
Luo et al. $[17]$ & 34 & 0.13 & 2.21 & 0.18 & 1.21 & - \\
Norberg [16] & 22 & 0.13 & 2.1 & - & - & - \\
Bearman \& Obasaju [15] & 22 & 0.13 & 2.1 & - & 1.2 & - \\
Lee [14] & 176 & - & 2.05 & $0.16-0.23$ & $0.68-1.32$ & - \\
Vickery [13] & 100 & 0.133 & 2.1 & - & - & - \\
\hline Minguez et al. $[20]$ & 21.4 & 0.141 & 2.2 & - & - & 1.28 \\
Ochoa \& Fueyo [33] & 21.4 & 0.139 & 2.01 & 0.22 & 1.4 & - \\
Sohankar et al. $[32]$ & 22 & $0.126-0.132$ & $2.03-2.32$ & $0.16-0.20$ & $1.23-1.54$ & - \\
Verstappen \& Veldman [35] & 22 & 0.133 & 2.09 & 0.178 & 1.45 & - \\
Rodi [31] & 22 & 0.13 & 2.3 & 0.14 & 1.15 & 1.46 \\
\hline LES results [29, 30] & 22 & $0.09-0.15$ & $2.02-2.77$ & $0.14-0.27$ & $1.15-1.79$ & $0.94-1.68$ \\
RANS results [29, 30] & 22 & $0.134-0.159$ & $1.64-2.43$ & $\approx 0-0.27$ & $0.31-1.49$ & $0.98-2.80$
\end{tabular}

Table 2: Comparison with previous experimental and numerical results of several bulk quantities. From left to right: Strouhal number, the time-averaged drag coefficient, the rms values of the fluctuations of the drag and lift coefficients, and the reattachment length. From top to bottom: the present DNS results, experimental results [13-20], LES results [20, 31-33] and the coarser DNS [35], and the range of values of LES and RANS results presented in the ERCOFTAC workshops [29, 30]. 

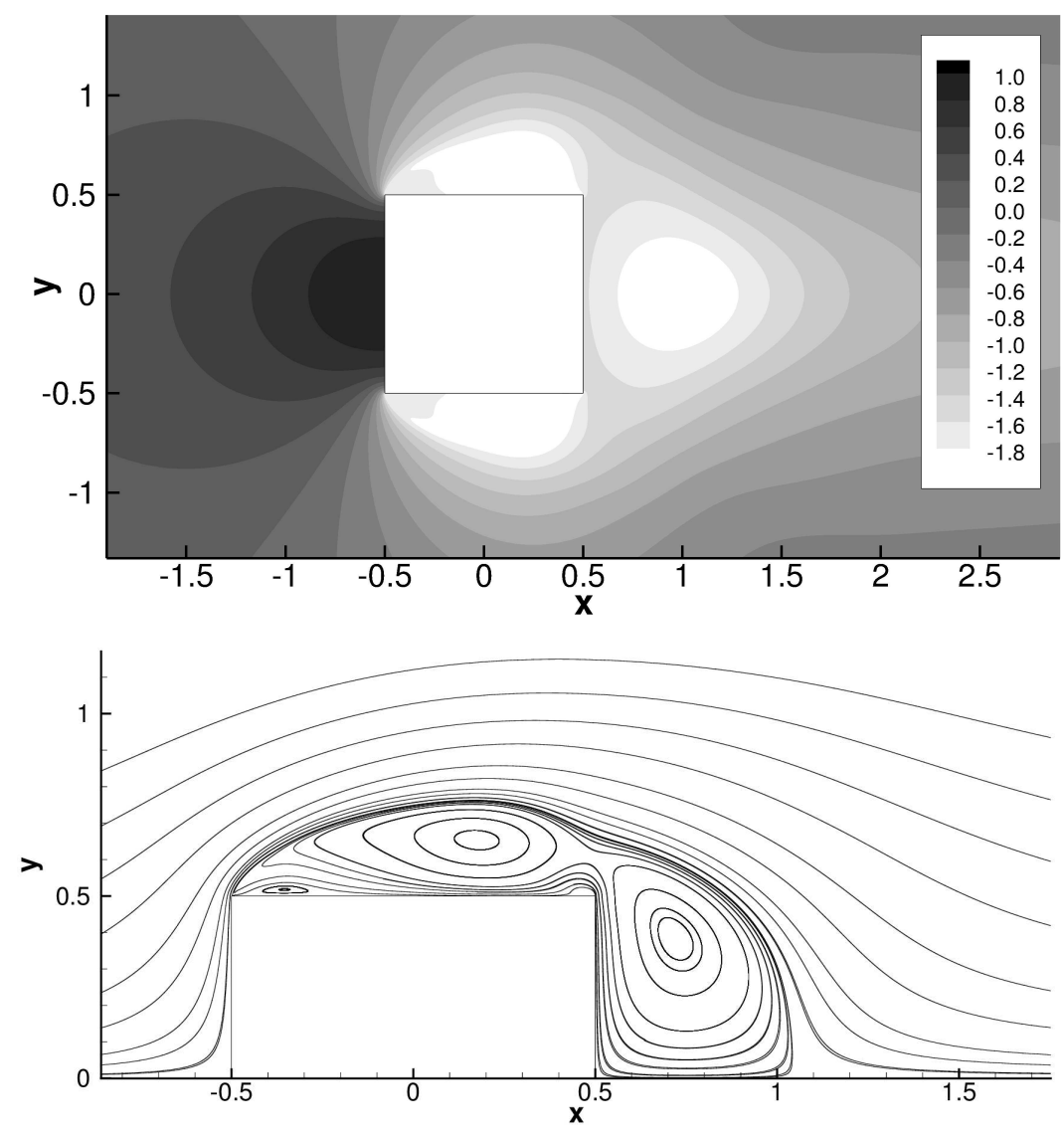

Figure 6: Averaged pressure field (top) and streamlines of the average flow (bottom).

sharp upstream corners force the flow to separate causing two large recirculations at the top and bottom areas of the cylinder. This strong curvature of the streamlines induces a strong pressure gradient. Then, the pressure value remains almost constant through the top and bottom walls until the effect of the downstream corners causes a slight increase of the pressure value. This pattern is clearly observed in Figure 7 (left) where the average pressure distribution around the obstacle is displayed. Moreover, streamlines show two smaller secondary recirculations near the upstream and downstream corners. 

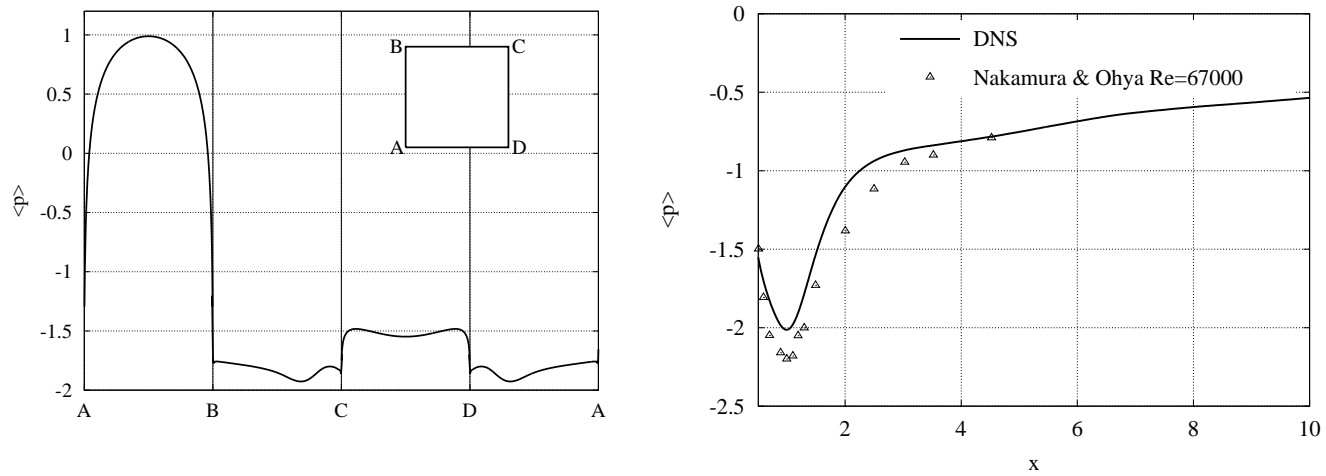

Figure 7: Left: averaged pressure distribution around the obstacle. Right: average pressure at domain centerline. Comparison with experimental results of Nakamura and Ohya [21].

They can be also identified with regions with positive wall-shear stress in Figure 3 (left). Finally, a pair of counter-rotating vortices is observed downstream the obstacle. They result from the shear-layer. Then, vortices of opposite sign roll up in an alternative manner resulting in the characteristic vortex shedding [1]. The intensity of the above-mentioned secondary recirculation near the downstream corner is strongly modulated by the phase of this shedding of vortices into the wake region (see Figure 13 and its corresponding movie). Pressure inside this wake region remains low with a minimum value of -2.01 on the centreline at around $x=1$. This can be clearly observed in Figure 7 (right) where time-averaged pressure, $\langle p\rangle$, at domain centerline is displayed together with the experimental results by Nakamura \& Ohya [21] at $R e=67000$. Although there is a very good agreement regarding the position of the peak its absolute value is under-predicted. According to the LES results by Sohankar [52] these differences cannot be attributed to Reynolds number effects. Nevertheless, current DNS results are significantly closer to 
the experimental data.

The drag and lift coefficients, $C_{D}$ and $C_{L}$, are obtained by integrating the pressure and the wall-shear stress on the surface of the cylinder. Notice that at this $R e$-number the latter is negligible respect to the pressure contribution. Their time-evolution for the last 100 time-units is displayed in Figure 12 (top). The averaged drag value, $\left\langle C_{D}\right\rangle=2.18$ is slightly higher than the experimental results shown in Table 2. Despite being a bulk quantity, it is quite difficult to predict. In [35], almost all the results from the ERCOFTAC workshops $[29,30]$ were collected. The numerical results showed a great dispersion with values of $\left\langle C_{D}\right\rangle$ from 1.9 to almost 2.8. Therefore, it was concluded that this flow forms a major challenge to LES techniques without finding a definite solution. There are many factors that can affect the prediction of the drag coefficient. Namely, the subgrid scale model, the grid resolution, the numerical schemes, the boundary conditions, the size of domain, etc. For instance, the artificial diffusion added by Upwind-like schemes may contaminate the results. In [35], they observed that significantly higher values of $\left\langle C_{D}\right\rangle$ are obtained for this type of schemes. As mentioned in the verification section, in this work we also observed that the size of the domain may play an important role probably due to blockage effects; therefore, two additional buffer areas of $20 D$ have been added at the top and bottom of the domain (see Figure 1 and Section 3). Another important source of errors can be caused if the three-dimensional effects are somehow restrained, e.g. the domain is too short in the span-wise direction or some unphysical effect is introduced by the subgrid scale model or the numerical method. A clear example thereof can be found in [53] where a 2D DNS simulation of 
the square cylinder at $R e=22000$ was carried out. Her results were also obtained with a symmetry-preserving discretization and similar spatial resolution in the stream-wise and cross-stream directions; however, in this case, the average drag coefficient was much lower, i.e. $\left\langle C_{D}\right\rangle=1.25$. From an experimental point-of-view, an accurate prediction of drag force is not trivial $[21,32]$. Firstly, the free-stream turbulent intensity affects all the results in a significant manner and, in particular, tend to reduce the drag [13, 14, 21]. Lyn et al. [19] and Minguez et al. [20] reported a free-stream turbulence level of $\approx 2 \%$ and $0.8 \%$, respectively, whereas for the rest of experimental studies shown in Table 2 it was $\leq 0.5 \%$. Secondly, the blockage effects need to be considered. Regarding this issue, the experimental results of Lyn et al. [19] and Minguez et al. [20] with blockages of $7 \%$ and 5\%, respectively, were not corrected. With similar blockage ratios the rest of the experiments shown in Table 2 made use of the classical Maskell method [54] to correct the blockage effects. Although these ratios are relatively low (in fact, they are below the recommended upper limit for the Maskell method) they certainly affect the measured drag coefficient. The combination of these two effects itself may explain the slightly higher mean drag coefficient in the DNS simulation. Apart from the time-averaged drag coefficient, other global parameters are also shown in Table 2. Namely, the Strouhal number, $S t$, the rms values of the fluctuations of the drag and lift coefficients, $C_{D}^{r m s}$ and $C_{L}^{r m s}$, respectively, and the reattachment length, $l_{R}$. The time-averaged lift coefficient, $\left\langle C_{L}\right\rangle$, is not shown; it should be zero by symmetry. In our case $\left\langle C_{L}\right\rangle \approx 0.002$, which indicates that results are well converged. The reattachment length indicates the length of the time-averaged separation region behind the cylinder (see 
also the streamlines of the averaged flow in Figure 6 and the averaged streamwise velocity in Figure 11). Again, all the previous numerical studies show a large dispersion and no results were explicitly reported in the experiments. However, the profiles of the averaged stream-wise velocity in the wake region suggest that the reattachment length in the experiment by Lyn et al. [19] is slightly longer than in our DNS results. An interpolation of their experimental results at $x=1$ and $x=2$ leads to $l_{R}=1.34$ whereas our reattachment length is $l_{R}=1.04$. The discussion about the $C_{D}^{r m s}$ and $C_{L}^{r m s}$ is left for the next section where the dynamics of the flow are discussed.

A more detailed description of the mean flow topology is given in Figures 8 and 9 where profiles of the time-averaged velocity in the near wall region are shown. Agreement with experimental data is in general good. The main differences are twofold. Firstly, the reverse flow observed in the vicinity of the wall near the upstream corner is less intense than in the experiments. Secondly, the boundary layer tends to grow slightly faster than in the experiments. The former is clearly observed in Figure 8 (top) where DNS results are compared with the experimental data by Lyn et al. [19] and Minguez et al. [20]. In the region closer to the wall, our results agree better with the results by Lyn et al. [19] whereas far from the wall they are between both sets of experimental data. With regard to the slightly faster growth of the boundary layer, this can be observed in Figure 9 where a set of profiles of the mean stream-wise and cross-stream velocity components are compared with the experimental results by Minguez et al. [20]. The same trend is also observed for the turbulent quantities displayed in Figure 10: although there is a very good agreement with the peak values, they are slightly shifted away 

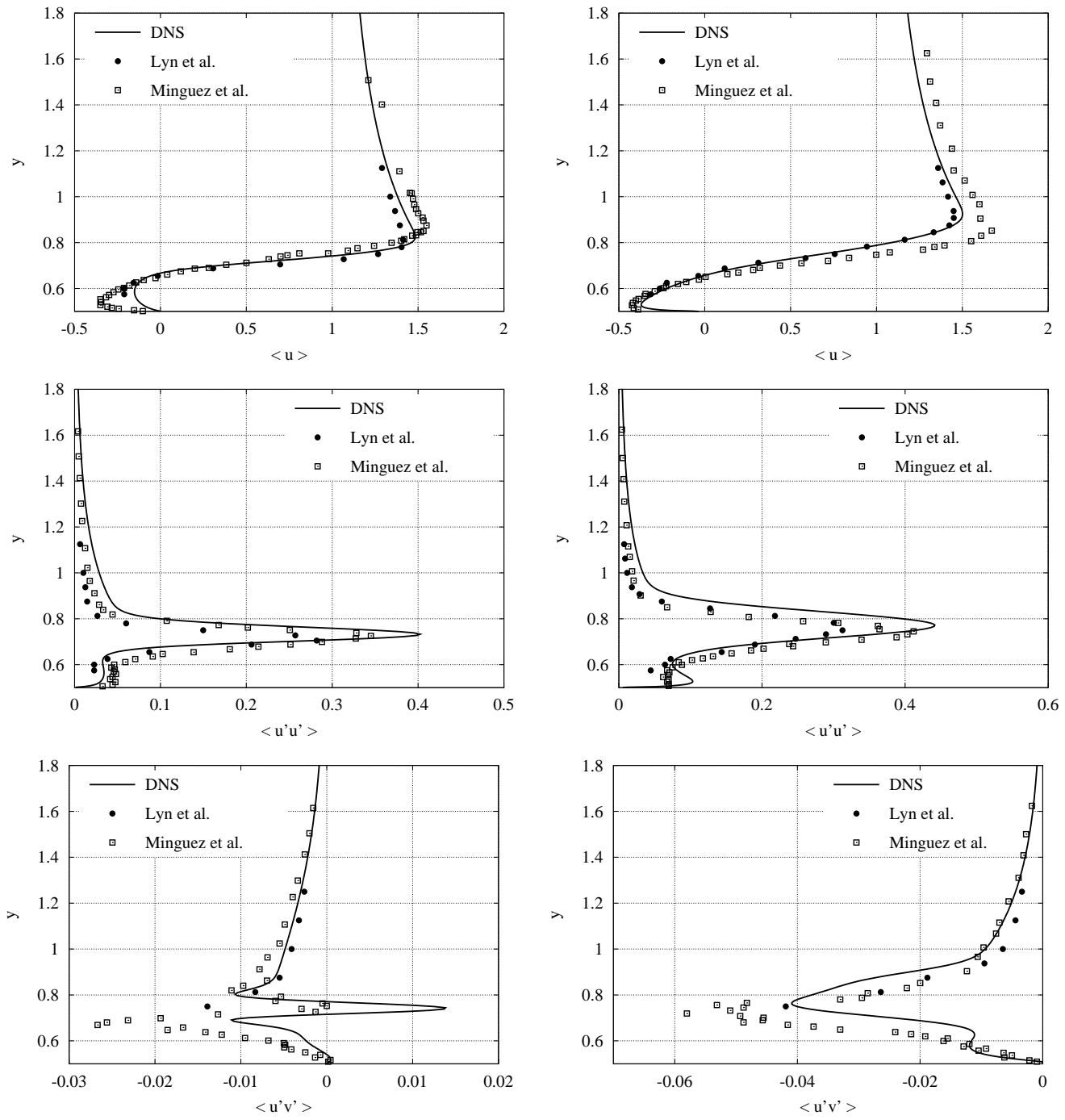

Figure 8: Profiles of the averaged stream-wise velocity, $\langle u\rangle$ (top), the stream-wise normal stress, $\left\langle u^{\prime} u^{\prime}\right\rangle$ (middle) and the shear stress component $\left\langle u^{\prime} v^{\prime}\right\rangle$ (bottom) at two different locations: $x=-0.125$ (left) and $x=0.125$ (right). Comparison with the experimental results of Lyn et al. [19] and Minguez et al. [20].

from wall. Regarding the wake region, comparison with the experimental data by Lyn et al. [19] in shown in Figure 11. In general, the averaged 

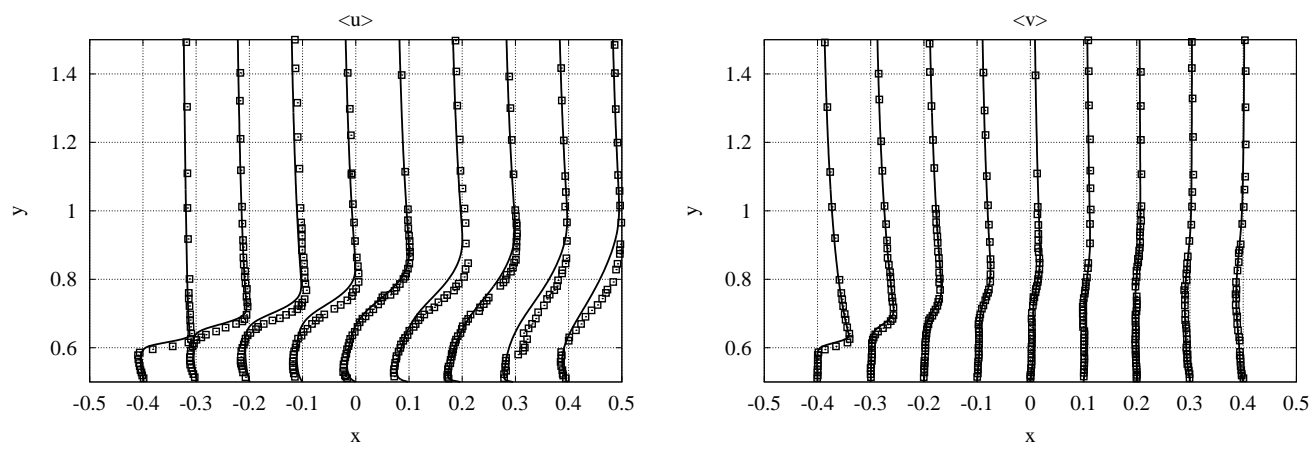

Figure 9: Profiles of the averaged stream-wise, $\langle u\rangle$ and the cross-stream velocities, $\langle v\rangle$ in the near cylinder region. Comparison with the experimental results of Minguez et al. [20].
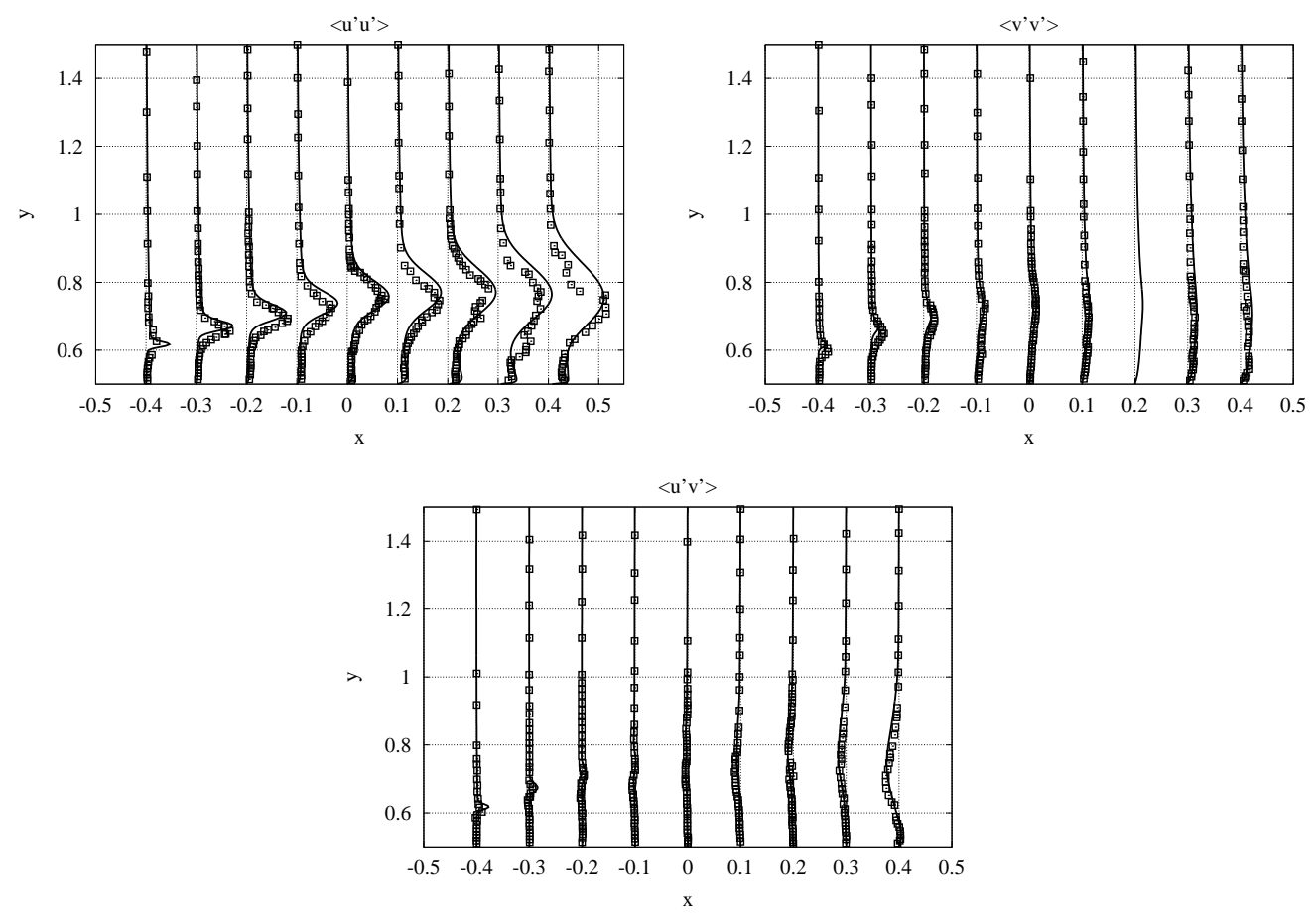

Figure 10: Profiles of the normal stresses $\left\langle u^{\prime} u^{\prime}\right\rangle$ and $\left\langle v^{\prime} v^{\prime}\right\rangle$, and the shear stress component $\left\langle u^{\prime} v^{\prime}\right\rangle$ in the near cylinder region. Comparison with the experimental results of Minguez et al. [20]. 

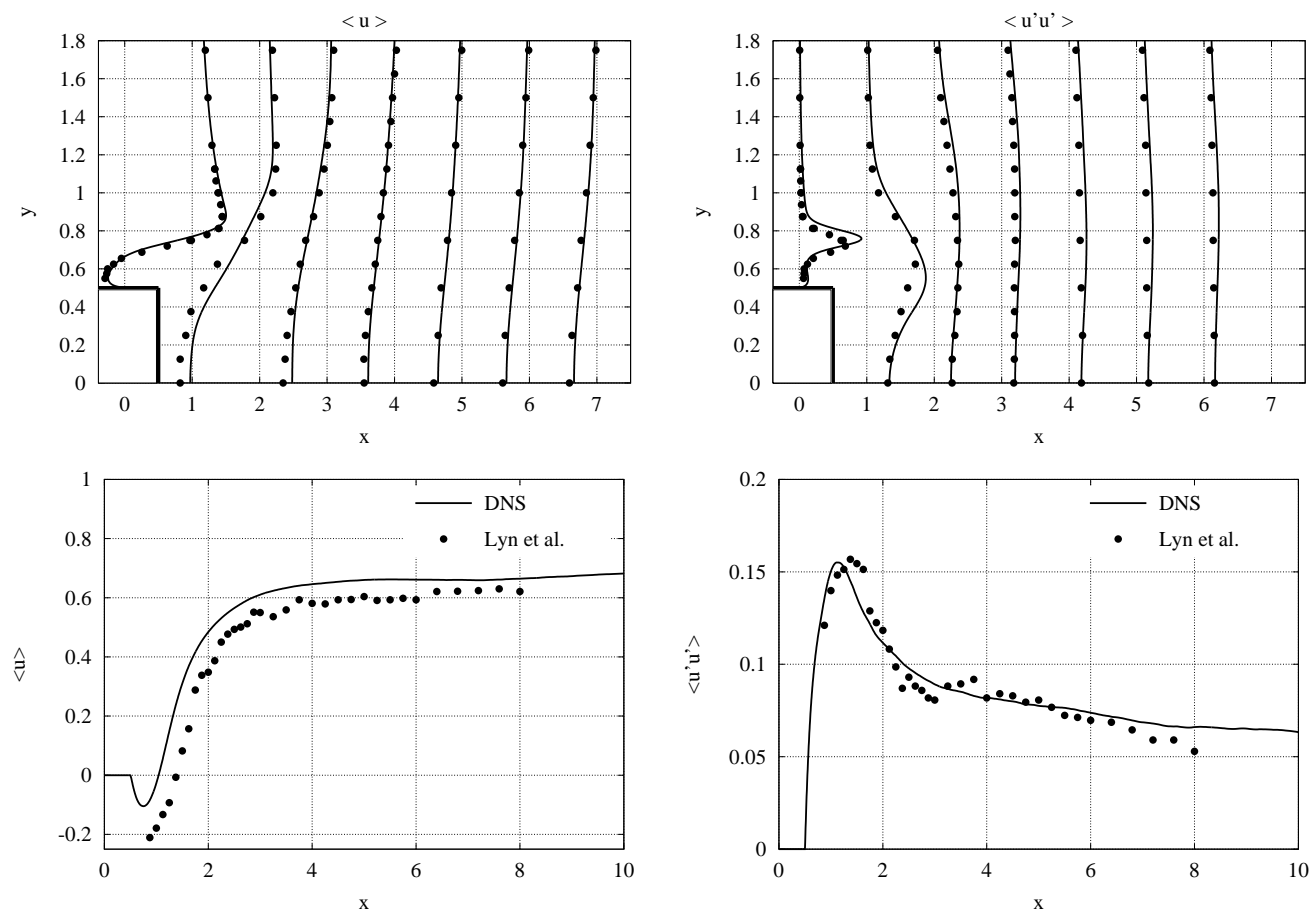

Figure 11: Top: profiles of the averaged stream-wise velocity, $\langle u\rangle$, and the stream-wise normal stress, $\left\langle u^{\prime} u^{\prime}\right\rangle$, in the wake region. Bottom: same quantities at domain centerline. Comparison with the experimental results of Lyn et al. [19].

stream-wise velocity profiles are in good agreement although slightly lower values around the $x$-axis were measured in the experiment. This difference remains approximately constant for all the wake region where the streamwise velocity at the domain centerline reaches a plateau with approximately a value of 0.66 (see Figure 11, bottom). This value is higher than the experimental data by Lyn et al. [19], which is about 0.6. Although the same trend is observed in LES studies [29, 32], values are significantly higher (up to approximately 0.9 in some cases) and a great disparity is observed among LES results. The most remarkable differences occur in the recirculation region 
where the magnitude of the stream-wise velocity is about twice lower than in the experiment (in the DNS the negative peak is located at $x=0.75$ with a value of -0.105). In this regard, very similar results have been reported in previous LES studies [29, 32].
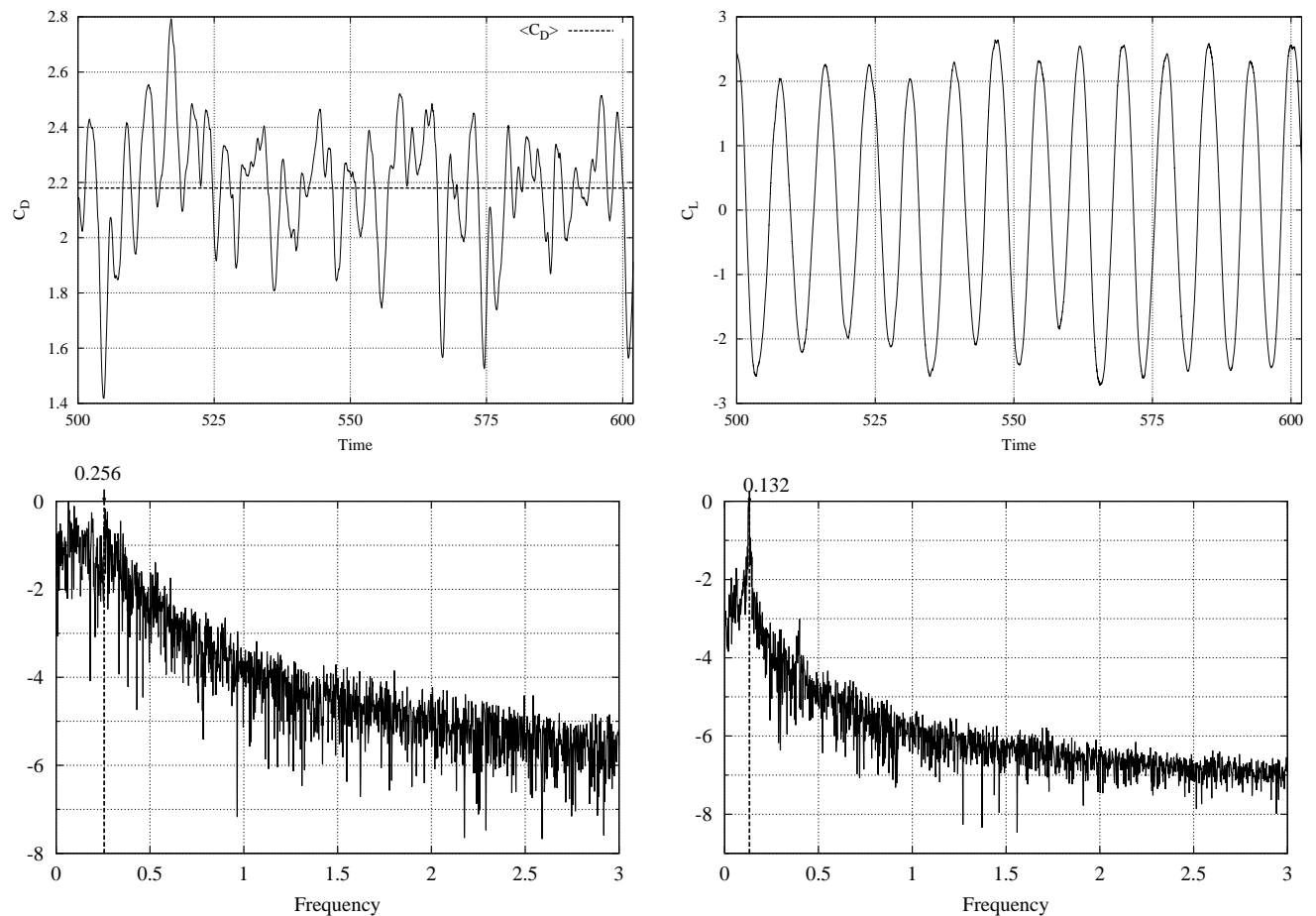

Figure 12: Top: time evolution of the drag (left) and lift (right) coefficients for the last 90 time-units. Bottom: normalized energy spectra of the drag (left) and lift (right) coefficients.

\subsection{Flow dynamics}

The most characteristic feature of this type of flow is the Von Kármán vortex shedding in the wake region (see Figure 13 and its corresponding movie). It results from the alternating formation of asymmetric vortices that results from the flow separation. This phenomenon induces forces on the cylinder. 


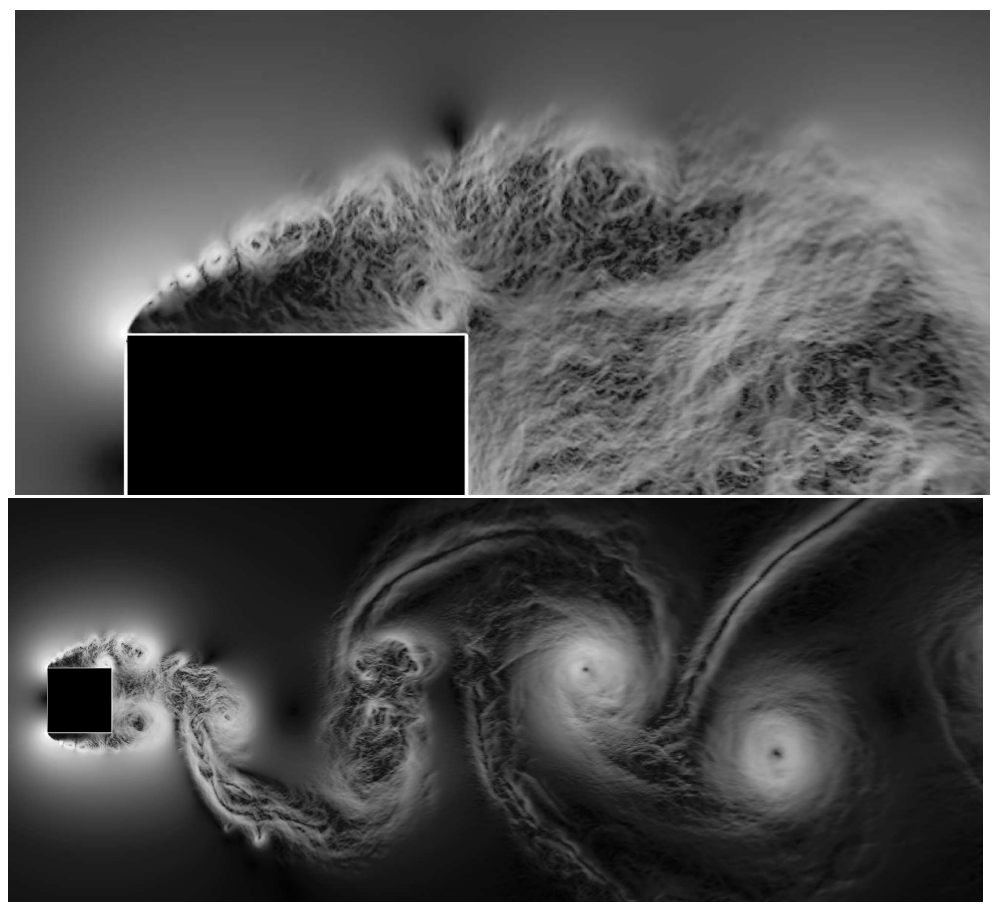

Figure 13: Visualization of the Kelvin-Helmholtz structures (top) and the Von Kármán vortex street (bottom). Instantaneous magnitude of the pressure gradient. See the movie attached in the supplementary material [36].
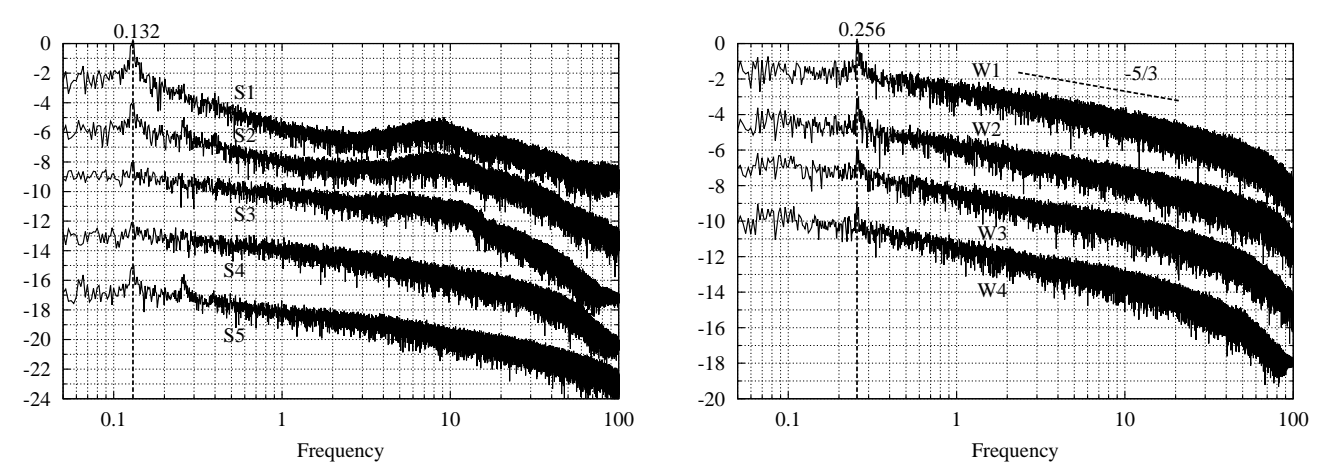

Figure 14: Normalized energy spectra of the stream-wise velocity at different locations. See Table 3 for details. For the sake of clarity, the energy spectra of consecutive points are shifted 4 (left) and 3 decades (right), respectively. 


\begin{tabular}{lrlc} 
Point & \multicolumn{1}{l}{$y$} & Dominant frequency \\
\hline S1 & -0.45 & 0.63 & 0.132 \\
S2 & -0.40 & 0.63 & 0.132 \\
S3 & -0.30 & 0.63 & 0.132 \\
S4 & 0.00 & 0.63 & - \\
S5 & 0.50 & 0.63 & 0.132 \\
\hline W1 & 1.00 & 0.00 & 0.256 \\
W2 & 1.50 & 0.00 & 0.256 \\
W3 & 2.50 & 0.00 & 0.256 \\
W4 & 3.50 & 0.00 & 0.256 \\
\hline
\end{tabular}

Table 3: List of monitoring locations.

Figure 12 (top) displays the time-evolution of the drag and lift coefficients for the last 100 time-units. The lift coefficient clearly shows a dominant frequency corresponding with the formation of such vortices. The peak of its normalized energy spectra (see Figure 12, bottom) is located at 0.132. As expected, this Strouhal number, St, is in very good agreement with the experimental data and most of the numerical studies (see Table 2). Regarding the amplitude of the fluctuations, $C_{L}^{r m s}=1.71$, is slightly above other numerical studies. As mentioned in Section 3, such differences in the numerical results could be easily attributed to insufficient time-integration periods (see Figure 5). Regarding the experimental results, Lee [14] provided results for $C_{L}^{r m s}$ for two different inflow conditions. Namely, 0.68 for a turbulent flow and 1.32 for a smooth flow. Such a range of values gives an idea of the difficulty of the problem: even low levels of turbulence intensity can sig- 
nificantly affect the results making the accurate matching between different experiments, and between experiments and numerical studies, a great challenge. Similar conclusions can be drawn for the time evolution of the drag coefficient and the measure of its fluctuations. However, in this case, there is not a clearly dominant frequency in its spectrum displayed in Figure 12 (bottom). Apart from a peak at the Strouhal frequency, $S t=0.132$, several harmonics and sub-harmonics are also observed; actually, the peak locates at 0.256 which corresponds to the second harmonic.

To study the dynamics of the flow with more detail the set of monitoring points given in Table 3 has been analyzed. The first five points are located in the shear layer zone whereas the rest correspond to the wake region. Actually, the first three points are the same points studied in [20]. They are located close to the upper corner. This region is characterized by the formation of small vortices in the shear layer due to the Kelvin-Helmholtz instability that are rapidly convected downstream. They can be observed in the instantaneous snapshot displayed in Figure 13 (see also its corresponding movie). The size of these vortices grow quickly triggering turbulence before they reach the downstream corner of the cylinder. Actually, they break up into finer structures before being engulfed into the much larger Von Kármán vortices. The first Kelvin-Helmholtz structure developed in the shear layer is observed at $x \approx-0.45$, in good agreement with the experiment results by Brun et al. [22]. This is analyzed quantitatively in Figure 14 where the normalized energy spectra for the stream-wise velocity component are displayed. The results corresponding to the shear layer region clearly show that the Von Kármán vortex shedding and the Kelvin-Helmholtz instability act 
at different frequencies. The former is the dominant frequency in the shear layer region (see also Table 3) except for the point S4 that does not displayed any dominant frequency. Notice that in this point the vortices related with the Kelvin-Helmholtz instability mechanism are already broken leading to a higher level of turbulence fluctuations and a clear broadening of the region where turbulent statistics are significant (see Figure 10 for details about several turbulent statistics). Finally, for the point S5 the Von Kármán frequency is again dominant. On the other hand, the Kelvin-Helmholtz instability is only observed for the first three monitoring points. In the location S3 it is observed a broadening of the energy distribution towards low frequencies leading to an almost flat energy spectra between the Von Kármán frequency, $f_{V K}$, and the Kelvin-Helmholtz frequency, $f_{K H}$. This backscattering effect is typically observed in the transition phase of turbulent shear flows. Regarding the value of the Kelvin-Helmholtz frequency, the correlation proposed by Prasad and Williamson [55] for circular cylinders, $f_{K H} / f_{V K}=0.0235 R e^{0.67}$, predicts a Kelvin-Helmholtz frequency equal to $\approx 2.5$. In our case, a relatively broad band frequency peak is observed around 8 . This value is in relatively good agreement with the experimental results obtained in [20] where a peak around 6 was measured. In our case this peak is not so visible probably due to a shorter integration period. However, the main difference is that in the experiments this peak was only observed in the point S2 indicating that the Kelvin-Helmholtz instability occurs earlier in the present DNS simulation. Similar conclusions were reached in [20] when comparing their LES results with the experimental data. Moreover, they suggested that the fact that in the experiments the Kelvin-Helmholtz instability is not observed in 
the point S3 implies that transition to turbulence occurs earlier. Nevertheless, the turbulent statistics displayed in Figure 10 do not seem to be affected by these discrepancies in the flow dynamics. The magnitude of the turbulent fluctuations is in very good agreement with the experimental data. The only significant difference is due to the above-mentioned fact that the boundary layer tends to grow slightly faster.

The dynamics of the wake region is mainly characterized by the Von Kármán vortex shedding. The normalized energy spectra of the four points located in this region (see Table 3) show essentially the same: the dominant frequency is 0.256 that approximately corresponds to the second harmonic of the Strouhal frequency. Then, as expected, an inertial range with the classical $-5 / 3$ slope extends up to point where physical dissipation becomes dominant. The inertial range tends to get shorter for more downstream positions indicating that the level of turbulence tends to decrease. This is also clearly observed in Figure 11 (right) where the stream-wise normal stress, $\left\langle u^{\prime} u^{\prime}\right\rangle$, in the wake region is compared with the experimental results of Lyn et al. [19]. Again there is a good agreement although slightly lower values were measured in the experiment. Similar to the average stream-wise velocity displayed in Figure 11 (left), this difference remains approximately constant for all the wake region.

\section{Concluding remarks}

A direct numerical simulation of the turbulent flow around a square cylinder at Reynolds number 22000 (based on the cylinder diameter and the inflow velocity) has been carried out on a Cartesian staggered mesh 
with $1272 \times 1174 \times 216$ grid points. A fully-conservative fourth-order spatial discretization has been used together with a second-order explicit timeintegration scheme. Special emphasis has been given to determine the proper domain size and time-integration period in the process to verify the simulation. In this regard, and compared with previous numerical studies, the domain has been substantially increased in the cross-stream direction whereas the span-wise direction has been slightly reduced. Moreover, the distance of the upstream face of the cylinder from the inflow has also been increased in order to obtain solutions independent of the domain size. Then, timeaveraged results have been presented and discussed together with the available experimental data. In the present simulation, the reverse flow near the upstream corner is less intense and the boundary layer tends to grow slightly faster. Apart from this, the agreement with the experimental data in the near wall region is rather good also for turbulent statistics. In the wake region, the average stream-wise velocity and the normal stress are also in rather good agreement although slightly lower values were measured in the experiment. These minor differences remain approximately constant for all the wake region. Finally, frequency analysis of velocity samples has been carried out to analyze both the Kelvin-Helmholtz vortical structures produced by the flow separation at the leading edge of the cylinder and the Von Kármán vortex shedding in the wake region. In the present simulation the Kelvin-Helmholtz instability is observed more downstream than in the experiments. This may suggest that in this case the transition to turbulence occurs later. However, the above-mentioned good agreement for the turbulent statistics in the near wall region indicates that transition is being well 
captured.

\section{Acknowledgments}

This work has been financially supported by the Ministerio de Economía y Competitividad, Spain (ENE2014-60577-R), the Russian Science Foundation (project 15-11-30039) and a Ramón y Cajal postdoctoral contract (RYC2012-11996). Calculations have been performed on the IBM MareNostrum supercomputer at the Barcelona Supercomputing Center. The authors thankfully acknowledge these institutions. We also thank the anonymous reviewers for their comments and remarks which helped to improve the quality of this work.

\section{References}

[1] J. H. Gerrard, The mechanics of the formation region of vortices behind bluff bodies, Journal of Fluid Mechanics 25 (1966) 401-413.

[2] M. Coutanceau, R. Bouard, Experimental determination of the main features of the viscous flow in the wake of a circular cylinder in uniform translation. Part 1: steady flow, Journal of Fluid Mechanics 79 (pt 2) (1977) 231-256.

[3] A. E. Perry, M. S. Chong, T. T. Lim, The vortex-shedding process behind two-dimensional bluff bodies, Journal of Fluid Mechanics 116 (1982) 77-90. 
[4] C. Norberg, Experimental investigation of the flow around a circular cylinder: influence of aspect ratio, Journal of Fluid Mechanics 258 (1994) $287-316$.

[5] L. Ong, J. Wallace, The velocity field of the turbulent very near wake of a circular cylinder, Experiments in Fluids 20 (6) (1996) 441-453.

[6] X. Ma, G. S. Karamanos, G. E. Karniadakis, Dynamics and lowdimensionality of a turbulent near wake, Journal of Fluid Mechanics 410 (2000) 29-65.

[7] P. Parnaudeau, J. Carlier, D. Heitz, E. Lamballais, Experimental and numerical studies of the flow over a circular cylinder at Reynolds number 3900, Physics of Fluids 20 (8) (2008) 085101.

[8] O. Lehmkuhl, I. Rodríguez, R. Borrell, A. Oliva, Low-frequency unsteadiness in the vortex formation region of a circular cylinder, Physics of Fluids 25 (8) (2013) 085109.

[9] M. Breuer, Large eddy simulation of the subcritical flow past a circular cylinder: Numerical and modeling aspects, International Journal for Numerical Methods in Fluids 28 (9) (1998) 1281-1302.

[10] A. G. Kravchenko, P. Moin, Numerical studies of flow over a circular cylinder at $\mathrm{ReD}=3900$, Physics of Fluids 12 (2) (2000) 403-417.

[11] J. Franke, W. Frank, Large eddy simulation of the flow past a circular cylinder at $\mathrm{ReD}=3900$, Journal of Wind Engineering and Industrial Aerodynamics 90 (10) (2002) 1191-1206. 
[12] G. Ghorbaniasl, V. Agnihotri, C. Lacor, A self-adjusting flow dependent formulation for the classical Smagorinsky model coefficient, Physics of Fluids 25 (5) (2013) 055102.

[13] B. J. Vickery, Fluctuating lift and drag on a long square cylinder of square cross-section in a smooth and in a turbulent stream, Journal of Fluid Mechanics 25 (1966) 481-494.

[14] B. E. Lee, The effect of turbulence on the surface pressure field of square prisms, Journal of Fluid Mechanics 69 (1975) 263-282.

[15] P. W. Bearman, E. D. Obasaju, Experimental study of pressure fluctuations on fixed and oscillating square-section cylinders, Journal of Fluid Mechanics 119 (1982) 297-321.

[16] C. Norberg, Flow around rectangular cylinders: Pressure forces and wake frequencies, Journal of Wind Engineering and Industrial Aerodynamics 49 (1-3) (1993) 187-196.

[17] S. C. Luo, M. G. Yazdani, Y. T. Chew, T. S. Lee, Effects of incidence and afterbody shape on flow past bluff cylinders, Journal of Wind Engineering and Industrial Aerodynamics 53 (3) (1994) 375-399.

[18] D. Lyn, W. Rodi, The flaping shear layer formed by flow separation from the forward corner of a square cylinder, Journal of Fluid Mechanics 267 (1994) 353-376.

[19] D. Lyn, S. Einav, W. Rodi, J. Park, A laser doppler velocimetry study of ensemble-averaged characteristics of the turbulent near wake of a square cylinder, Journal of Fluid Mechanics 304 (1995) 285-319. 
[20] M. Minguez, C. Brun, R. Pasquetti, E. Serre, Experimental and highorder LES analysis of the flow in near-wall region of a square cylinder, International Journal of Heat and Fluid Flow 32 (3) (2011) 558-566.

[21] Y. Nakamura, Y. Ohya, Effects of turbulence on the mean flow past two-dimensional rectangular cylinders, Journal of Fluid Mechanics 149 (1984) 255-273.

[22] C. Brun, S. Aubrun, T. Goossens, P. Ravier, Coherent structures and their frequency signature in the separated shear layer on the sides of a square cylinder, Flow, Turbulence and Combustion 81 (1-2) (2008) $97-114$.

[23] M. Breuer, J. Bernsdorf, T. Zeiser, F. Durst, Accurate computations of the laminar flow past a square cylinder based on two different methods: lattice-Boltzmann and finite-volume, International Journal of Heat and Fluid Flow 21 (2000) 186-196.

[24] A. Sohankar, C. Norberg, L. Davidson, Low-Reynolds-number flow around a square cylinder at incidence: study of blockage, onset of vortex shedding and outlet boundary condition, International Journal for Numerical Methods in Fluids 26 (1999) 39-56.

[25] A. Sohankar, C. Norberg, L. Davidson, Simulation of three-dimensional flow around a square cylinder at moderate Reynolds numbers, Physics of Fluids 11 (1999) 288-306.

[26] A. K. Saha, G. Biswas, K. Muralidhar, Three-dimensional study of flow 
past a square cylinder at low Reynolds numbers, International Journal of Heat and Fluid Flow 24 (1) (2003) 54-66.

[27] A. Saha, Unsteady flow past a finite square cylinder mounted on a wall at low Reynolds number, Computers and Fluids 88 (2013) 599-615.

[28] M. Zhao, L. Cheng, T. Zhou, Numerical simulation of vortex-induced vibration of a square cylinder at a low Reynolds number, Physics of Fluids 25 (2) (2013) 023603.

[29] W. Rodi, J. H. Ferziger, M. Breuer, M. Pourquié, Status of large eddy simulation: Results of a workshop, Journal of Fluids Engineering, Transactions of the ASME 119 (2) (1997) 248-262.

[30] P. R. Voke, Flow past a square cylinder: test case LES2, in: J. C. et al. (Ed.), Direct and Large Eddy Simulation II, 1997, pp. 355-373.

[31] W. Rodi, Comparison of LES and RANS calculations of the flow around bluff bodies, Journal of Wind Engineering and Industrial Aerodynamics 6971 (1997) 55-75.

[32] A. Sohankar, L. Davidson, C. Norberg, Large eddy simulation of flow past a square cylinder: Comparison of different subgrid scale models, Journal of Fluids Engineering, Transactions of the ASME 122 (1) (2000) $39-47$.

[33] J. S. Ochoa, N. Fueyo, Large eddy simulation of the flow past a square cylinder, in: PHOENICS User Conference 2004, Melbourne, Australia, 2004 . 
[34] R. W. C. P. Verstappen, A. E. P. Veldman, Direct Numerical simulation of turbulence at lower costs, Journal of Engineering Mathematics 32 (1997) 143-159.

[35] R. W. C. P. Verstappen, A. E. P. Veldman, Spectro-consistent discretization of Navier-Stokes: a challenge to RANS and LES, Journal of Engineering Mathematics 34 (1998) 163-179.

[36] The DNS results presented in this paper are publicly available in http://www.cttc.upc.edu/downloads/SqCyl22K.

[37] R. W. C. P. Verstappen, A. E. P. Veldman, Symmetry-Preserving Discretization of Turbulent Flow, Journal of Computational Physics 187 (2003) 343-368.

[38] A. J. Chorin, Numerical Solution of the Navier-Stokes Equations, Journal of Computational Physics 22 (1968) 745-762.

[39] F. X. Trias, O. Lehmkuhl, A self-adaptive strategy for the timeintegration of Navier-Stokes equations, Numerical Heat Transfer, part B 60 (2) (2011) 116-134.

[40] A. Gorobets, F. X. Trias, A. Oliva, A parallel MPI+OpenMP+OpenCL algorithm for hybrid supercomputations of incompressible flows, Computers \& Fluids 88 (2013) 764-772.

[41] J. B. Perot, An analysis of the fractional step method, Journal of Computational Physics 108 (1993) 51-58. 
[42] S. Armfield, R. Street, The Fractional-Step Method for the NavierStokes Equations on Staggered Grids: The Accuracy of Three Variations, Journal of Computational Physics 153 (2) (1999) 660-665.

[43] S. Armfield, R. Street, An analysis and comparison of the time accuracy of fractional-step methods for the Navier-Stokes equations on staggered grids, International Journal for Numerical Methods in Fluids 38 (3) (2002) 255-282.

[44] F. X. Trias, O. Lehmkuhl, A. Oliva, C.D. Pérez-Segarra, R.W.C.P. Verstappen, Symmetry-preserving discretization of Navier-Stokes equations on collocated unstructured meshes, Journal of Computational Physics 258 (2014) 246-267.

[45] J. Kim, P. Moin, Application of a Fractional-Step Method to Incompressible Navier-Stokes Equations, Journal of Computational Physics 123 (1985) 308-323.

[46] F. X. Trias, M. Soria, A. Oliva, C. D. Pérez-Segarra, Direct numerical simulations of two- and three-dimensional turbulent natural convection flows in a differentially heated cavity of aspect ratio 4, Journal of Fluid Mechanics 586 (2007) 259-293.

[47] S. Murakami, W. Rodi, S. Mochida, S. Sakamoto, Large-eddy simulation of turbulent vortex-shedding flow past 2D square cylinder, in: ASME Fluids Engineering Division Summer Meeting, Washington DC, USA, 1993. 
[48] P. Moin, K. Mahesh, Direct numerical simulation: A Tool in Turbulence Research, Annual Review of Fluid Mechanics 30 (1998) 539-578.

[49] G. Grötzbach, Revisiting the resolution requirements for turbulence simulations in nuclear heat transfer, Nuclear Engineering and Design 241 (11) (2011) 4379-4390.

[50] A. W. Vreman, J. G. M. Kuerten, Comparison of direct numerical simulation databases of turbulent channel flow at $R e_{\tau}=180$, Physics of Fluids 26 (1) (2014) 015102.

[51] H. Zhang, F. X. Trias, A. Gorobets, A. Oliva, Direct numerical simulation of a fully developed turbulent square duct flow up to $R e_{\tau}=1200$, International Journal of Heat and Fluid Flow 54 (2015) 258-267.

[52] A. Sohankar, Flow over a bluff body from moderate to high Reynolds numbers using large eddy simulation, Computers and Fluids 35 (10) (2006) 1154-1168.

[53] A. Valcarce, Estudi de fluxes convectius en base a tècniques de CFD\&HT, Master thesis, Technical University of Catalonia, Spain (October 2012).

[54] E. C. Maskell, A theory of the blockage effects on bluff bodies and stalled wings in a closed wind tunnel, Reports and Memoranda, Aeronautical Research Council (ARC) (1963) 3400.

[55] A. Prasad, C. H. Williamson, The instability of the shear layer separating from a bluff body, Journal of Fluid Mechanics 333 (1997) 375-402. 